\title{
Transcalvarial and Transdural Involvement of Skull Actinomycosis with Recurrence
}

\author{
Kramadhari Harshith ${ }^{1}$ Krishnan Nagarajan ${ }^{1}$ Andi Sadayandi Ramesh ${ }^{2}$ Debasis Gochhait ${ }^{3}$
}

${ }^{1}$ Department of Radio-Diagnosis, Jawaharlal Institute of Postgraduate Medical Education and Research (JIPMER), Pondicherry, India

${ }^{2}$ Department of Neurosurgery, Jawaharlal Institute of Postgraduate Medical Education and Research (JIPMER), Pondicherry, India

${ }^{3}$ Department of Pathology, Jawaharlal Institute of Postgraduate Medical Education and Research (JIPMER), Pondicherry, India

\author{
Address for correspondence Krishnan Nagarajan, MD, DM, Department \\ of Radio-Diagnosis, JIPMER, Pondicherry 605006 \\ (e-mail: Iknagarajan1@gmail.com).
}

Indian J Neurosurg 2019;8:185-187

Abstract
Keywords
- actinomycosis
- transcalvarial
involvement
- transdural spread
- magnetic resonance
imaging
- histopathologic
diagnosis

Actinomycosis is a suppurative disease that involves chronic infection of the soft tissue and bone, caused by anaerobic gram-positive bacteria of Actinomyces genus, most commonly due to the organism Actinomyces israelii. These organisms are either anaerobic or microaerophilic, producing branching filaments resembling fungi. They were previously mistaken for fungi due to their capability to form filaments. The clinical infection can resemble several other chronic disorders such as tuberculosis or malignancy. Appropriate diagnosis and management are of paramount importance as the disease is treatable. Manifestations of the disease are generally in the form of abscess formation associated with complications such as fibrosis and discharging sinuses. The infection spreads diffusely throughout the soft tissue across the fascial barriers.

A 35-year-old woman presented with complaints of subacute onset of weakness of the left upper and lower limbs. The patient previously had history of headache for the previous few months with a diffuse swelling over the scalp. She was recently diagnosed with diabetes mellitus. There was no history of fever, and on examination, sensorium was normal. Power in the left upper limb and lower limb was $2 / 5$. Reflexes

received

July 26, 2018

accepted

October 5, 2018

published online

September 20, 2019 were brisk, and plantar reflexes were extensor. The random blood sugar was elevated. Blood urea, serum creatinine, and electrolytes were normal. The patient's hemoglobin was normal, and blood count showed mild eosinophilia (7\%). She was referred for imaging evaluation of her symptoms. Routine noncontrast and contrast-enhanced computed tomography was done, which showed a hyperdense lesion over the right parietal lobe associated with dural thickening. There was sclerosis in the overlying skull bone with irregularities in the

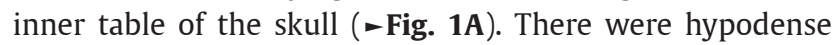
areas within the brain adjacent to the lesion. Minimal postcontrast enhancement was noted.

Contrast-enhanced magnetic resonance imaging (MRI) was done for further characterization of the lesion. MRI showed a large $\mathrm{T} 1$ isointense, $\mathrm{T} 2$ hypointense lesion in the over the right parietal lobe in the epidural space infiltrating into the brain parenchyma causing confluent FLAIR hyperintensities in the subcortical, deep, and periventricular white matter in the right cerebral hemisphere due to cerebritis or meningitis with vasogenic edema. It was associated with midline shift to the left side and apposition of the lateral 


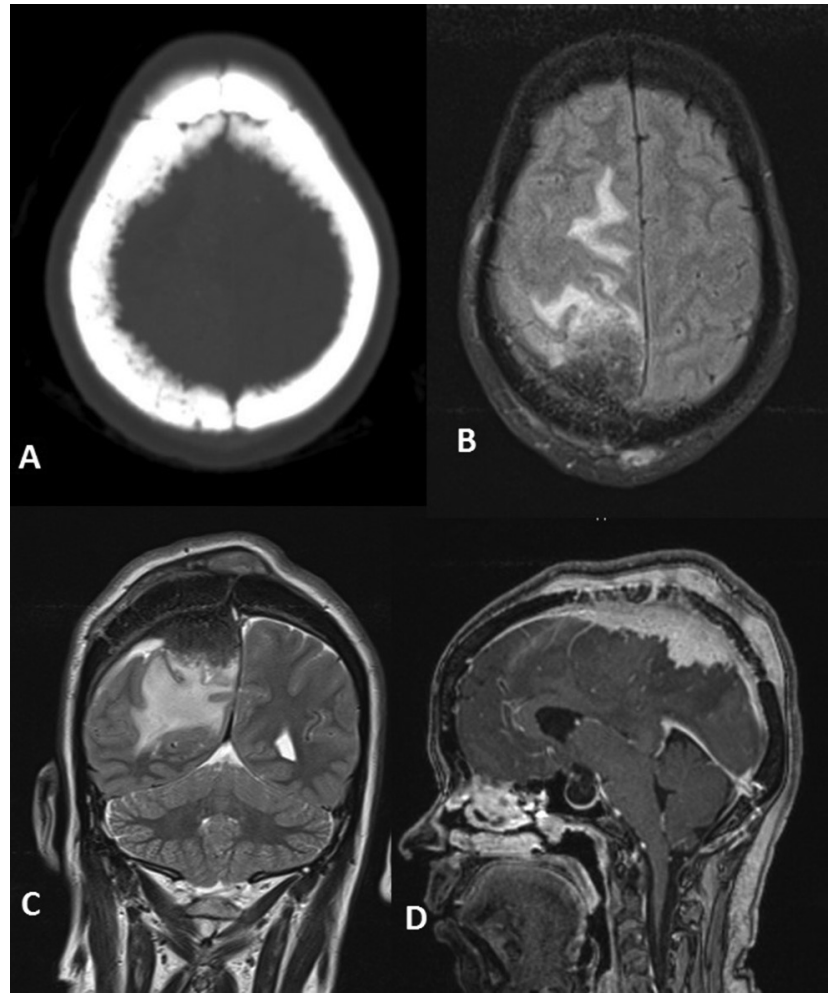

Fig. 1 Plain CT axial section (A) at the level of parietal convexity shows diffuse calvarial thickening and bone erosions along the inner table of the skull. FLAIR axial (B) and T2-weighted coronal (C) magnetic resonance images showing scalp, diploic and dural hypointense lesion infiltrating into brain parenchyma causing hyperintense edema. (D) Contrast-enhanced T1-weighted sagittal magnetic resonance image showing intense enhancement within the lesion and adjacent dura seen extending into the skin and subcutaneous tissue.

ventricles. Altered signal changes were noted in the diploic spaces with thickening of the parietal bone. An extracranial component was also noted, which was communicating with the intracranial lesion through the sagittal suture. Moderate postcontrast enhancement was noted in the lesion. Superior sagittal sinus was infiltrated by the lesion ( - Fig. 1B-D).

The initial imaging differential diagnoses were en plaque meningioma, tuberculosis, metastasis, and lymphoma due to the presence of soft tissue lesion along with underlying bony sclerosis and erosion of the inner table. Biopsy was obtained from the lesion as was subjected to histopathologic and microbiologic examination. Fungal microscopy and cultures were negative. The histopathologic examination was suggestive of actinomycosis. Right parietal craniotomy was done after the first MRI, and the lesion was debrided. On opening the skull, there was a mass with grayish black color that was submitted for frozen section with differential of a primary melanocytic lesion of the meninges to categorize the type of lesion. The frozen section showed granulomatous inflammation with some organisms that could not be categorized and was reported as inflammatory lesion likely to be a mycetoma, without any evidence of any neoplastic lesion.

On paraffin processing the tissue, the sections showed numerous well-formed granulomas ( - Fig. 2) with filamentous organisms in the center surrounded by a lymphohistiocytic collection and giant cell reaction. In addition, there

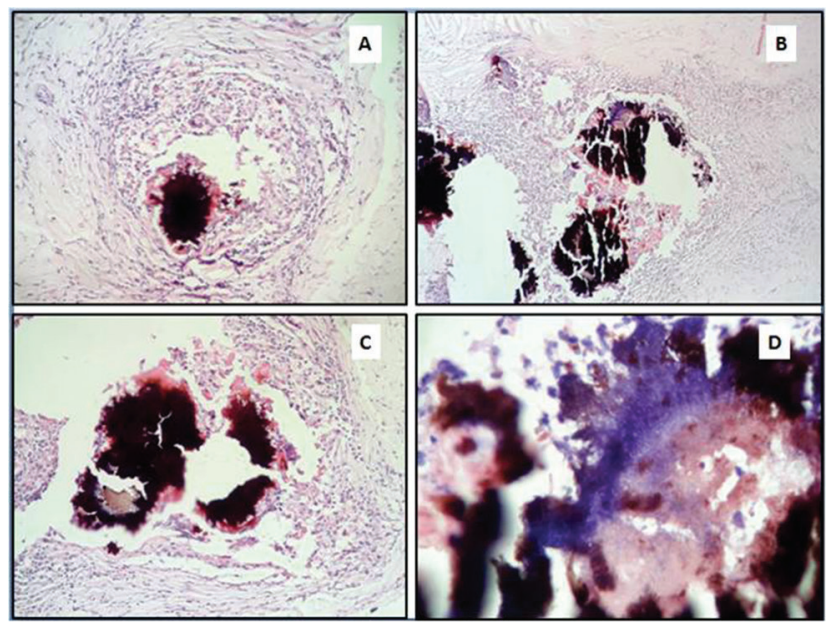

Fig. 2 Low-power images $(\mathbf{A}-\mathbf{C})$ showing the distinct well-formed granulomas along with the pigments and "Splendore-Hoeppli" material. (D) Oil immersion image highlighting the bluish/hematoxyphilic filamentous bacterial profiles.

was a distinct "Splendore-Hoeppli phenomenon" in the form of a nice annular eosinophilic to pinkish material around the bacterial organisms representing host response in the form of an antigen-antibody reaction. The higher-power images highlighted the bluish hematoxyphilic filamentous bacterial profile. No fungal organism was seen even on the special stains. With these findings, final diagnosis of $A$. israelii was made.

The patient was started on penicillin after the biopsy reports were available. Follow-up MRI done 5 months later revealed residual/recurrent lesion of smaller size than preoperative lesion. A second follow-up MRI performed 1.5 years after surgery showed persistence and mild increase in the size of the lesion compared with the second MRI. The patient was continued on antibiotics with plan for surgical decompression if symptomatically worsened.

The very first report of central nervous system (CNS) actinomycosis was described in 1889 by Delepine. ${ }^{1}$ Many isolated cases have been reported both in the preimaging era and after the advent of CT/MRI.-6 The CNS involvement is rare, and in the CNS, the common presentation is that of focal lesion as an abscess or simulating a mass. Studies using angiography have shown that arteritis is caused when there is subarachnoid extension similar to other infections such as tuberculosis. ${ }^{2}$

The CNS infection is generally secondary to a source in the respiratory tract, abdomen, pelvis, or cervicofacial routes such as dental or paranasal sinus manipulation and posttraumatic events. The source of CNS infection spreads through hematogenous routes. However, direct spread of the infection from dental infections, or from paranasal sinuses or other sites of cervicofacial region is also possible.

The type of CNS involvement is predominantly either focal lesion or dural/epidural involvement with adjacent parenchymal involvement. ${ }^{2-5}$ Though dura is considered a relative barrier, indolent infections such as tuberculosis are known to cause dural involvement. Local spread and invasion across tissue planes is considered one of the characteristic features of actinomycosis as seen across pleura in thoracic 
involvement, deep fascial planes of the neck in cervicofacial type, and dura in the CNS. ${ }^{6}$

Few case reports have described mainly the second type of dural/epidural actinomycosis with adjacent parenchymal involvement. ${ }^{3-5,7-12}$ The first report of an epidural actinomycotic infection was described by Kirsch and Stears in 1970 and hypothesized it due to spread from an indolent dental/ mandibular focus. ${ }^{3}$ In the two cases of cranial actinomycosis reported by Sundaram et al in 2004, one had an underlying penetrating injury, ${ }^{8}$ but other reports of calvarial/dural involvement did not show any obvious predisposition of either dental source of infection or trauma for the initiation of infection. However, actinomycosis has been ascribed to be the cause of mandibular osteoradionecrosis and this is considered to be due to preferential localization of actinomycosis to devitalized tissue as reported in thorax and in relatively less vascular mandible after radiotherapy. ${ }^{13,14}$ It is possible that actinomycosis may become indolent once it establishes in a relatively avascular ischemic milieu. Similar involvement of relatively less vascular mastoid and petrous temporal bone involvement have been described. ${ }^{9}$ The associated actinomycosis-induced arteritis might prolong the indolent course by the involvement of both scalp vessels and dural-side arteries giving rise to relatively avascular environment. This may explain the chronicity and recurrence that is encountered with actinomycosis.

The bacterial cultures can take long time to yield results and are positive in less than $50 \%$ of cases. Medical management is the primary mode of treatment for actinomycosis, and penicillin is the drug of choice. However, the duration of treatment can be as long as few months, and serial radiologic follow-up is necessary to assess resolution of the lesion. Inadequate treatment can result in relapses. Adequate surgical debridement will help control the infection and can shorten the duration of antibiotic treatment.

In our case, the patient was a recently diagnosed case of diabetes mellitus who presented with subacute onset of neurologic deficits on the left side. The patient had a history of chronic headache for the past 1 year with a scalp swelling. She had no other extracranial source of infection in the body. Uncontrolled diabetes with a state of chronic hyperglycemia causes changes in microenvironment of the tissue with altered wound healing and control of infection. Hence adequate control with oral hypoglycemic agents is necessary for the control of ongoing infections in the patients. Craniotomy and debridement with antibiotic therapy initially caused symptomatic improvement. However, the patient had a recurrence of the lesion on follow-up, which is known to occur in actinomycosis.

In conclusion, actinomycotic osteomyelitis of the calvarium is rare but a known complication of actinomycosis. The disease has a chronic course and generally presents with nonspecific symptoms. In imaging it can masquerade as either malignancy or other chronic diseases. A high clinical suspicion and adequate histopathologic examination of the lesion are important for diagnosis and follow-up of the patient. The patient will generally require debridement of the lesion with medical management for long durations for complete resolution. Even with adequate treatment, recurrences are known to occur.

\section{Conflicts of Interest}

None declared.

\section{Source of Support}

None.

\section{Acknowledgment}

None.

\section{References}

1 D'Ewart J, Dawson GD. Actinomycosis in the brain of a child. $\mathrm{Br}$ Med J 1927;1(3458):718-719

2 Wickbom GI, Davidson AJ. Angiographic findings in intracranial actinomycosis. A case report and consideration of pathogenesis. Radiology 1967;88(3):536-537

3 Kirsch WM, Stears JC. Actinomycotic osteomyelitis of the skull and epidural space. Case report. J Neurosurg 1970;33(3):347-351

4 Funaki B, Rosenblum JD. MR of central nervous system actinomycosis. AJNR Am J Neuroradiol 1995;16(5):1179-1180

5 N'Dri Oka D, Ba Zeze V, Varlet G, Boni N, Broalet E, Boukassa L. [Craniocerebral actinomycosis] [in French]. Rev Neurol (Paris) 1999;155(12):1082-1083

6 Wong VK, Turmezei TD, Weston VC. Actinomycosis. BMJ 2011;343:d6099

7 King AD, Chan YL, Wong KS, Sung JJ, Fung K, Poon WS. Cranial actinomycosis. Singapore Med J 1998;39(10):465-467

8 Sundaram C, Purohit AK, Prasad VS, Meena AK, Reddy JJ, Murthy JM. Cranial and intracranial actinomycosis. Clin Neuropathol 2004;23(4):173-177

9 Budenz CL, Tajudeen BA, Roehm PC. Actinomycosis of the temporal bone and brain: case report and review of the literature. Ann Otol Rhinol Laryngol 2010;119(5):313-318

10 Nomura M, Shin M, Ohta M, Nukui Y, Ohkusu K, Saito N. Atypical osteomyelitis of the skull base and craniovertebral junction caused by. Actinomyces. infection-case report. Neurol Med Chir (Tokyo) 2011;51(1):64-66

11 Mohindra S, Savardekar A, Rane S. Intracranial actinomycosis: varied clinical and radiologic presentations in two cases. Neurol India 2012;60(3):325-327

12 Roopesh Kumar VR, Madhugiri VS, Gundamaneni SK, Verma SK. Actinomycotic osteomyelitis of the cranial vault presenting with headache: an unusual presentation. BMJ Case Rep 2014;2014:bcr2013202501

13 Mabeza GF, Macfarlane J. Pulmonary actinomycosis. Eur Respir J 2003;21(3):545-551

14 Hansen T, Kunkel M, Kirkpatrick CJ, Weber A. Actinomyces in infected osteoradionecrosis--underestimated? Hum Pathol 2006;37(1):61-67 